Case Report

\title{
Cardiopulmonary Failure Requiring ECMO Bypass Resulting from Leukemia Cell Lysis in a Patient with Childhood Acute Myelomonocytic Leukemia
}

\author{
Michael Huang, ${ }^{1}$ Erin Owen, ${ }^{2}$ Scott Myers, ${ }^{1}$ and Ashok Raj ${ }^{1}$ \\ ${ }^{1}$ Division of Pediatric Hematology, Oncology and Stem Cell Transplantation, University of Louisville, Louisville, KY, USA \\ ${ }^{2}$ Division of Pediatric Critical Care Medicine, University of Louisville, Louisville, KY, USA \\ Correspondence should be addressed to Michael Huang; michael.huang@louisville.edu
}

Received 9 March 2015; Accepted 11 May 2015

Academic Editor: Salah Aref

Copyright ( 2015 Michael Huang et al. This is an open access article distributed under the Creative Commons Attribution License, which permits unrestricted use, distribution, and reproduction in any medium, provided the original work is properly cited.

Background. Childhood AML patients are at increased risk for early fatal pulmonary complications. Pulmonary leukostasis and systemic inflammatory response syndrome (SIRS) following leukemia cell lysis are the likely etiologies. Observation. Soon after initiation of AML chemotherapy, an 18-month-old female who met SIRS criteria sustained cardiopulmonary failure requiring ECMO support. Upon recovery, the patient went on to complete therapy and remains in remission without permanent neurologic or cardiac sequelae. Conclusion. Cytokine release syndrome from rapid cell lysis was the likely cause as infectious workup failed to reveal a definitive etiology and drug hypersensitivity testing to the chemotherapy agents was negative.

\section{Background}

Patients with childhood acute myelogenous leukemia (AML) are at increased risk for early death secondary to pulmonary complications. Pulmonary leukostasis and systemic inflammatory response syndrome (SIRS) following leukemia cell lysis have been implicated as the likely etiologies. We herein report the case of an 18-month-old female who sustained cardiopulmonary failure requiring Extracorporeal Membrane Oxygenation (ECMO) support following initiation of chemotherapy for acute myelomonocytic (M4eo) leukemia.

\section{Case Report}

The patient was an 18-month-old female with AML M4eo. She presented with fever and left calf cellulitis and was placed on IV clindamycin. Her $\mathrm{CBC}$ at the time showed circulating blasts with WBC $17.51 \times 10^{9} / \mathrm{L}, \mathrm{Hgb} 9.2 \mathrm{~g} / \mathrm{dL}$, and platelets $88 \times 10^{9} / \mathrm{L}$. Bone marrow aspiration showed $15 \%$ myeloblasts with increased eosinophils. Cytogenetic testing and immunotyping by fluorescent in situ hybridization (FISH) revealed inversion of chromosome 16 , as well as $7 \mathrm{q}$ deletion in a small $(5.7 \%)$ population of blasts. She was discharged with her cellulitis improved and then readmitted a week later to begin chemotherapy. On readmission, her CBC showed WBC $18.9 \times 10^{9} / \mathrm{L}$, Hgb $8.5 \mathrm{~g} / \mathrm{dL}$, and platelets $60 \times 10^{9} / \mathrm{L}$, with $4 \%$ circulating blasts noted. The patient was started on an induction regimen of cytarabine, daunorubicin, and etoposide per the COG AAML0531 protocol.

On day 4 from initiation of chemotherapy, our patient developed fever $\left(\operatorname{Tmax} 39.7^{\circ} \mathrm{C}\right)$, tachycardia $(169 / \mathrm{min})$, and tachypnea $(100 / \mathrm{min})$. Her WBC had dropped to $3.59 \times$ $10^{9} / \mathrm{L}$ from $11.01 \times 10^{9} / \mathrm{L}$ the day before. Serial chemistries, creatinine, and uric acid all remained within normal limits throughout. A chest X-ray at that time showed a patchy right middle lobe infiltrate concerning for pneumonia. Empiric antimicrobial coverage was instituted with IV ceftriaxone and vancomycin. Over the subsequent $24 \mathrm{hrs}$, the patient's respiratory status progressively deteriorated requiring transfer to the Pediatric Intensive Care Unit (PICU) for escalation of respiratory support. Chemotherapy was stopped at this time and antibiotic coverage was expanded with the addition of meropenem and caspofungin. Initially the patient was placed on high flow nasal cannula at 10 liters per minute with inhaled nitric oxide at 20 parts per million but quickly required intubation for ongoing oxygenation failure and respiratory 
acidosis. With positive pressure ventilation, the patient developed hemodynamic compromise with significant metabolic and lactic acidoses requiring dopamine and epinephrine continuous infusions. Due to ongoing hypoxia and hypercarbia, the patient was transitioned to high frequency oscillatory ventilation (HFOV) within 5 hours of initial intubation. Within one hour after placement on HFOV, the patient suffered a prolonged cardiopulmonary arrest (intermittent but persistent for approximately 2 hours) requiring compressions and multiple doses of epinephrine, calcium chloride, and sodium bicarbonate. The patient was ultimately placed on venoarterial ECMO by the general surgical team due to ongoing cardiopulmonary failure. Arterial blood gas measurement just prior to arrest showed $\mathrm{pH}$ 6.91, $\mathrm{pCO}_{2}$ 76, $\mathrm{pO}_{2} \quad 53, \mathrm{HCO}_{3} 15$, base excess -21 , and $\mathrm{O}_{2}$ sat $62 \%$. This yielded a $\mathrm{paO}_{2}: \mathrm{FiO}_{2}$ ratio of 53 and an oxygenation index of 49 , both consistent with acute respiratory distress syndrome (ARDS). Chest radiograph obtained showed bilateral diffuse pulmonary opacification. Following the episode of cardiopulmonary arrest, the patient went into nonoliguric renal failure with doubling of creatinine $(0.8 \mathrm{mg} / \mathrm{dL})$ and development of hyperphosphatemia $(12.2 \mathrm{mg} / \mathrm{dL})$ and was placed on continuous venovenous hemodialysis (CVVHD) via the ECMO circuit.

Our patient had completed 6 of the planned 10 days of chemotherapy and the decision was made to defer the remainder of her first course of induction due to multiorgan failure. She had RSV upper respiratory infection on presentation at initial diagnosis 3 weeks before. A repeat respiratory viral panel PCR at the time of admission for initiation of induction chemotherapy was still positive for RSV. PCR from bronchoalveolar lavage (BAL) fluid at the time of respiratory compromise, however, tested negative for RSV. The remainder of her infectious workup failed to show an etiologic organism. The patient had a continuous electroencephalogram during days 1 through 4 of ECMO that demonstrated potential hypoxic-ischemic injury. Her echocardiogram (ECHO) performed while the patient was on ECMO showed elevated right-sided pressures (half systemic) but with normal structure, function, and systolic ejection fraction (LVEF 48.3\%), similar to an ECHO obtained 24 hours prior to her arrest. She was weaned off both ECMO and CVVHD by day 9 and extubated by day 13. Hypersensitivity testing for both cytarabine and etoposide yielded negative results. Her leukemia was in remission by morphology, flow, and FISH at the end of induction I. She did not suffer any substantial or permanent neurologic sequelae from the cardiopulmonary arrest. Her second course of induction was given in the ICU with no resulting adverse events. She went on to receive the remainder of her chemotherapy cycles and remains in leukemia remission.

\section{Discussion}

Cytokine-release syndrome as a result of leukemia cell lysis has been previously elucidated as a possible mechanism behind early pulmonary complications seen in newly diagnosed AML patients [1-3]. It is speculated that cytokines known to be secreted by myeloblasts are released after cell lysis, either spontaneously or after initiation of chemotherapy, and exert their effect at the level of the lung endothelial cell [4]. Many myeloblast cell lines constitutively express proinflammatory cytokines not found in normal hematopoietic cells such as IL-1 beta, IL-6, IL-8, and TNF-alpha [5-7]. IL-1 beta and TNF-alpha have been identified in bronchoalveolar lavage fluids from patients with acute respiratory distress syndrome (ARDS) [8], an acute inflammatory process in the airspaces, and lung parenchyma that is not dissimilar to the lysis pneumopathy described in AML patients.

Similar to previous reports, the clinical picture in our patient was consistent with SIRS (fever, tachycardia, and respiratory distress) and occurred during rapid tumor reduction [3]. There were 2 notable differences, however, namely, (1) the severity of pulmonary compromise and (2) leukemia remission achieved in our patient despite cessation of treatment midinduction. Although our patient was positive for RSV on viral PCR testing by nasal swab, BAL testing was negative for RSV. As such, the positive result was felt to be due to ongoing viral shedding, which is not uncommon in patients undergoing chemotherapy. None of the previously described cases were severe enough to result in cardiac arrest and need for ECMO bypass. The presence of elevated rightsided pressures with an otherwise normal ECHO 24 hours prior to her arrest and while on ECMO led us to conclude that our patient's cardiac compromise was secondary to primary respiratory failure rather than from direct cardiotoxicity. While the patient did develop metabolic and lactic acidoses in the hours leading up to her arrest, this is most likely a result of anaerobic metabolism at the end-organ level due to profound hypoxemia associated with ARDS as evidenced by her $\mathrm{paO}_{2}: \mathrm{FiO}_{2}$ ratio and oxygenation index just prior to her arrest. Furthermore, the introduction of positive pressure ventilation followed by HFOV was a contributing factor. By converting the intrathoracic cavity from a negative to a positive pressure chamber, more than likely the patient's overall hemodynamic status was acutely stressed with a drop in right-sided venous return to the heart as well as a potential abrupt increase in her pulmonary vascular resistance. This is in addition to the pulmonary hypertension she was exhibiting with right ventricular pressures half of her systemic systolic pressure. Though cytarabine has been reported to cause anaphylaxis [9] and proinflammatory cytokine release at higher doses [10], our patient had a negative skin test anergy and went on to receive subsequent cytarabine doses without further complications.

As this cell lysis-related phenomenon can occur during early chemotherapy for AML, particularly in M4/M4eo/M5 patients [3], vigilance with close monitoring is needed once induction treatment is started. Steroids with their added ability to induce leukemia cell apoptosis may be beneficial but their role needs to be clearly defined. Future studies looking at the cytokine expression profile during this phenomenon may be beneficial in identifying other candidate anti-inflammatory agents (e.g., tocilizumab, etanercept).

Unlike previously reported cases, our patient's symptoms did not improve quickly enough to allow resumption of induction therapy. Despite this, our patient went on to achieve complete hematologic and cytogenetic remission at 
the end of induction I. One possible explanation would be an antileukemia effect secondary to cytokine proliferation. In fact, several studies have demonstrated the potential role of cytokines in the treatment of AML [11]. The proinflammatory molecule IL-2, which enhances tumor cytotoxicity by inducing proliferation and activation of T and NK cells, is the cytokine that has been most extensively studied in adult as well as childhood AML $[12,13]$. Though a rare phenomenon, various case reports have reported spontaneous remission of AML following episodes of infection [14-17], suggesting that triggered $\mathrm{T}$ cell-driven immune responses likely via cytokine activation can induce myeloid leukemia cell remission. More specifically, cytokines such as TNF and IL-2, both of which are released during active infections, have been suggested to play a potential role in inducing spontaneous AML remission $[18,19]$.

\section{Conflict of Interests}

The authors declare that there is no conflict of interests regarding the publication of this paper.

\section{References}

[1] T. S. K. Wan, S. F. Yip, Y. M. Yeung, L. C. Chan, and S. K. $\mathrm{Ma}$, "Fatal diffuse alveolar damage complicating acute myeloid leukemia with abnormal eosinophils and trisomy X," Annals of Hematology, vol. 81, no. 3, pp. 167-169, 2002.

[2] F. Perez-Zincer, J. V. Juturi, E. D. Hsi, G. A. Hoeltge, L. A. Rybicki, and M. E. Kalaycio, "A pulmonary syndrome in patients with acute myelomonocytic leukemia and inversion of chromosome 16," Leukemia and Lymphoma, vol. 44, no. 1, pp. 103-109, 2003.

[3] N. Hijiya, M. L. Metzger, S. Pounds et al., "Severe cardiopulmonary complications consistent with systemic inflammatory response syndrome caused by leukemia cell lysis in childhood acute myelomonocytic or monocytic leukemia," Pediatric Blood and Cancer, vol. 44, no. 1, pp. 63-69, 2005.

[4] A. Stucki, A.-S. Rivier, M. Gikic, N. Monai, M. Schapira, and O. Spertini, "Endothelial cell activation by myeloblasts: molecular mechanisms of leukostasis and leukemic cell dissemination," Blood, vol. 97, no. 7, pp. 2121-2129, 2001.

[5] D. Bradbury, G. Bowen, R. Kozlowski, I. Reilly, and N. Russell, "Endogenous interleukin-1 can regulate the autonomous growth of the blast cells of acute myeloblastic leukemia by inducing autocrine secretion of GM-CSF," Leukemia, vol. 4, no. 1, pp. 44-47, 1990.

[6] W. Oster, N. A. Cicco, H. Klein et al., "Participation of the cytokines interleukin 6, tumor necrosis factor-alpha, and interleukin 1-beta secreted by acute myelogenous leukemia blasts in autocrine and paracrine leukemia growth control," The Journal of Clinical Investigation, vol. 84, no. 2, pp. 451-457, 1989.

[7] A. Tobler, B. Moser, B. Dewald et al., "Constitutive expression of interleukin- 8 and its receptor in human myeloid and lymphoid leukemia," Blood, vol. 82, no. 8, pp. 2517-2525, 1993.

[8] R. B. Goodman, J. Pugin, J. S. Lee, and M. A. Matthay, "Cytokine-mediated inflammation in acute lung injury," Cytokine \& Growth Factor Reviews, vol. 14, no. 6, pp. 523-535, 2003.

[9] M. Markham, S. B. Howell, M. King, C. Pfeifle, and S. I. Wasserman, "Anaphylactic reaction to cytarabine: in vitro evidence that the response is immunoglobulin E mediated," Medical and Pediatric Oncology, vol. 12, no. 3, pp. 201-203, 1984.

[10] T. Ek, M. Jarfelt, L. Mellander, and J. Abrahamsson, "Proinflammatory cytokines mediate the systemic inflammatory response associated with high-dose cytarabine treatment in children," Medical and Pediatric Oncology, vol. 37, no. 5, pp. 459-464, 2001.

[11] E. Ferretti, C. Cocco, I. Airoldi, and V. Pistoia, "Targeting acute myeloid leukemia cells with cytokines," Journal of Leukocyte Biology, vol. 92, no. 3, pp. 567-575, 2012.

[12] G. Meloni, R. Foa, S. Capria et al., "IL-2 for the treatment of acute leukemias," Leukemia, vol. 6, no. 2, pp. 28-30, 1992.

[13] B. J. Lange, F. O. Smith, J. Feusner et al., "Outcomes in CCG2961, a Children's Oncology Group phase 3 trial for untreated pediatric acute myeloid leukemia: a report from the Children's Oncology Group," Blood, vol. 111, no. 3, pp. 1044-1053, 2008.

[14] N. Jain, J. Hubbard, F. Vega, G. Vidal, G. Garcia-Manero, and G. Borthakur, "Spontaneous remission of acute myeloid leukemia: report of three cases and review of the literature," Clinical Leukemia, vol. 2, no. 1, pp. 64-67, 2008.

[15] C. Fozza, S. Bellizzi, S. Bonfigli, P. M. Campus, F. Dore, and M. Longinotti, "Cytogenetic and hematological spontaneous remission in a case of acute myelogenous leukemia," European Journal of Haematology, vol. 73, no. 3, pp. 219-222, 2004.

[16] O. Maywald, D. Buchheidt, J. Bergmann et al., "Spontaneous remission in adult acute myeloid leukemia in association with systemic bacterial infection-case report and review of the literature," Annals of Hematology, vol. 83, no. 3, pp. 189-194, 2004.

[17] C. I. Müller, M. Trepel, R. Kunzmann, A. Lais, R. Engelhardt, and M. Lübbert, "Hematologic and molecular spontaneous remission following sepsis in acute monoblastic leukemia with translocation (9;11): a case report and review of the literature," European Journal of Haematology, vol. 73, no. 1, pp. 62-66, 2004.

[18] M. Mitterbauer, M. Fritzer-Szekeres, G. Mitterbauer et al., "Spontaneous remission of acute myeloid leukemia after infection and blood transfusion associated with hypergammaglobulinaemia," Annals of Hematology, vol. 73, no. 4, pp. 189-193, 1996.

[19] P. Musto, G. D’Arena, L. Melillo et al., "Spontaneous remission in acute myeloid leukaemia: a role for endogenous production of tumour necrosis factor and interleukin-2?" British Journal of Haematology, vol. 87, no. 4, pp. 879-880, 1994. 


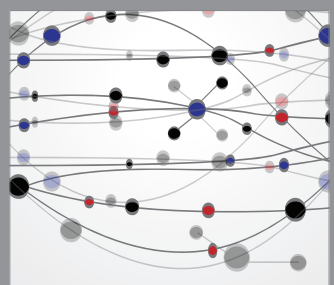

The Scientific World Journal
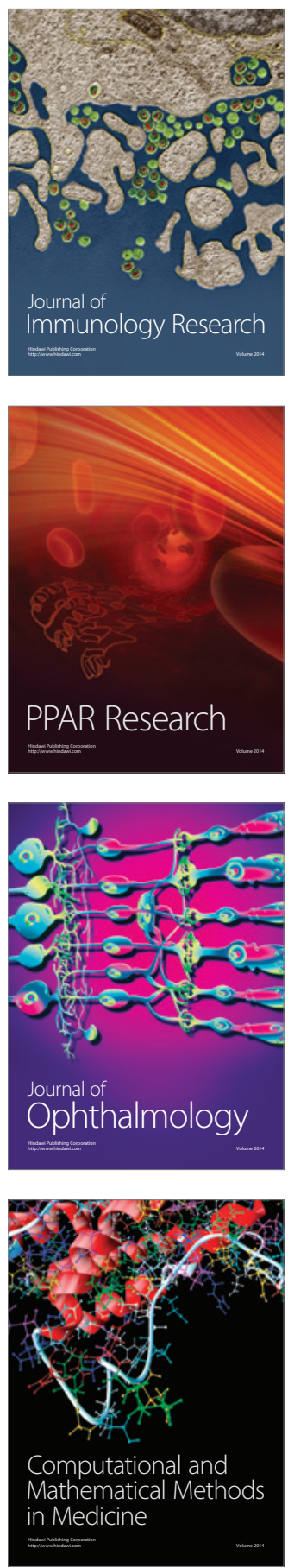

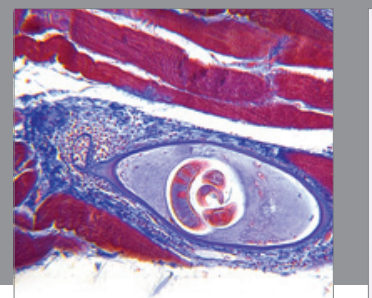

Gastroenterology

Research and Practice
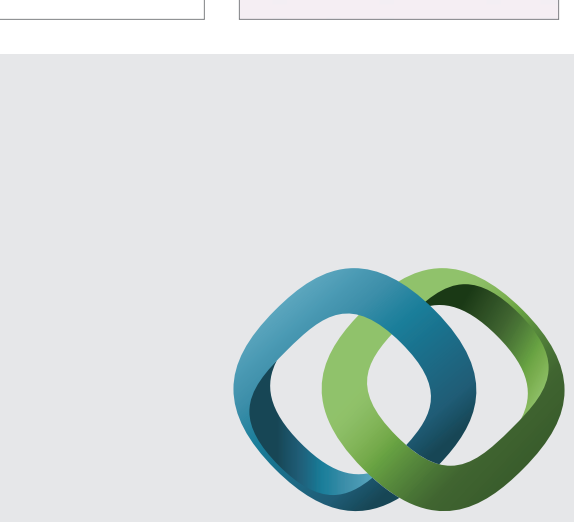

\section{Hindawi}

Submit your manuscripts at

http://www.hindawi.com
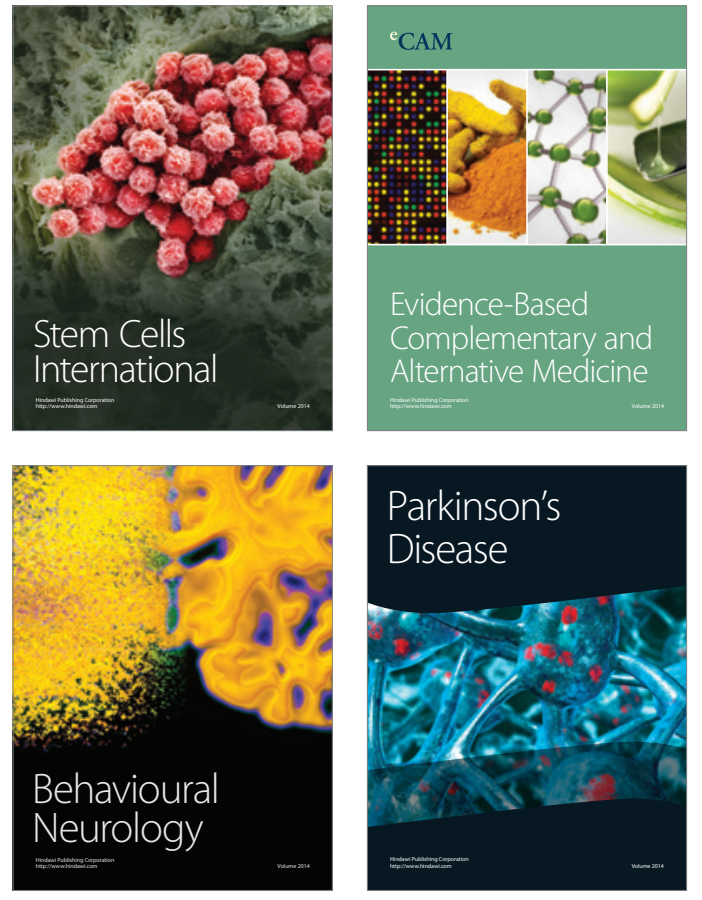
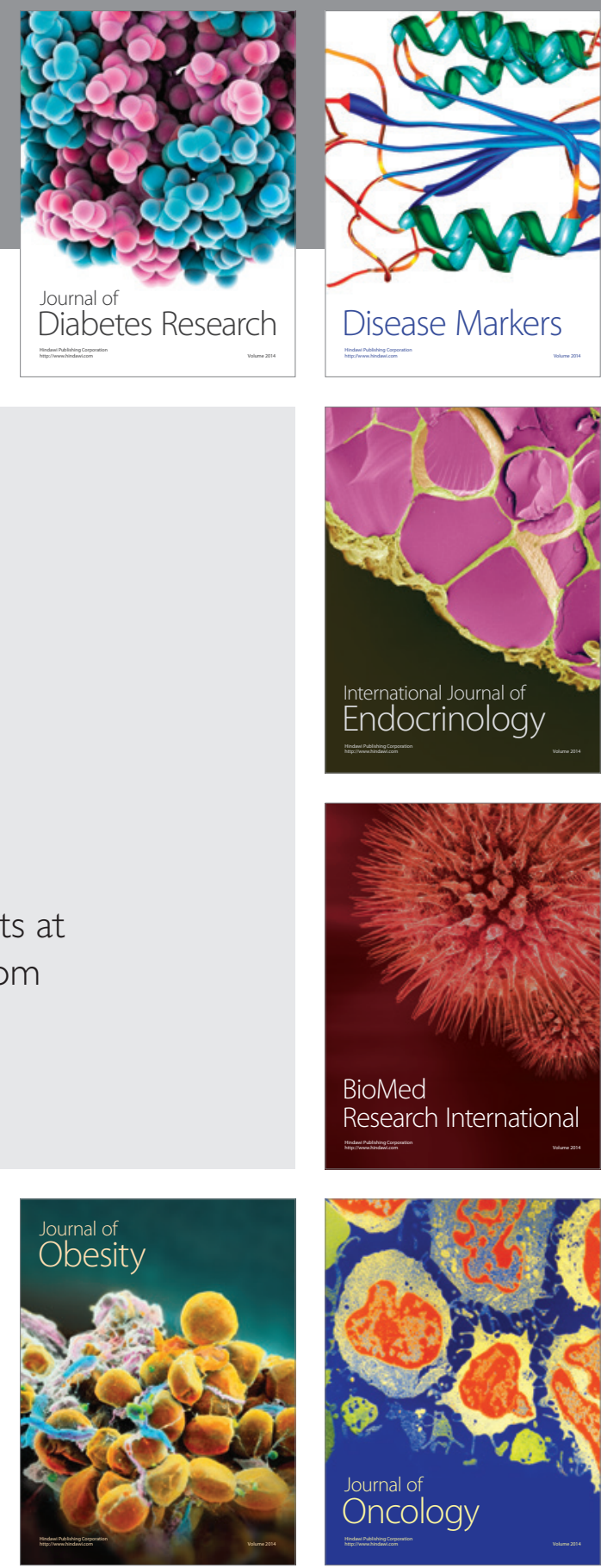

Disease Markers
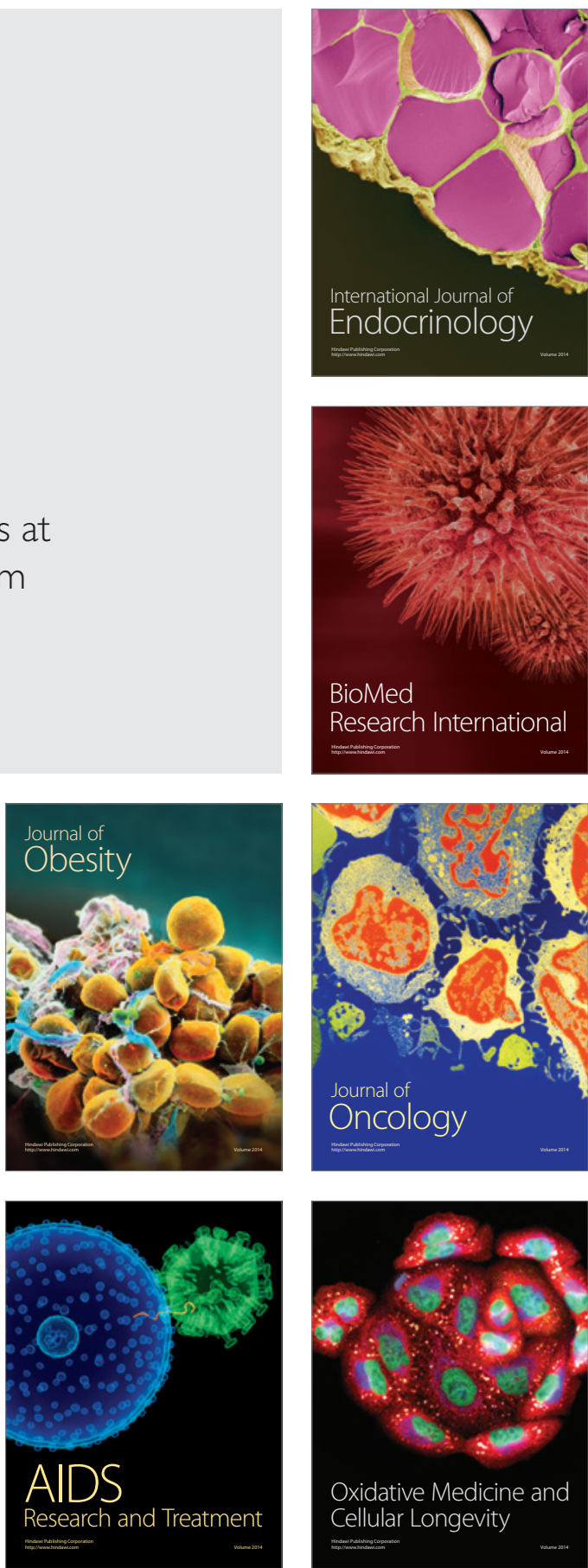